\title{
Anti-Plasmodium falciparum invasion ligand antibodies in a low malaria transmission region, Loreto, Peru
}

Elizabeth Villasis ${ }^{1 \dagger}$, Mary Lopez-Perez ${ }^{2 \dagger}$, Katherine Torres $^{1}$, Dionicia Gamboa ${ }^{1,3}$, Victor Neyra ${ }^{1}$, Jorge Bendezu ${ }^{1}$, Nancy Tricoche ${ }^{2}$, Cheryl Lobo ${ }^{4}$, Joseph M Vinetz ${ }^{1,3,5^{*}}$ and Sara Lustigman ${ }^{2^{*}}$

\begin{abstract}
Background: Erythrocyte invasion by Plasmodium falciparum is a complex process that involves two families; Erythrocyte Binding-Like (EBL) and the Reticulocyte Binding-Like (PfRh) proteins. Antibodies that inhibit merozoite attachment and invasion are believed to be important in mediating naturally acquired immunity and immunity generated by parasite blood stage vaccine candidates. The hypotheses tested in this study were 1) that antibody responses against specific $P$. falciparum invasion ligands (EBL and $P f R h$ ) differ between symptomatic and asymptomatic individuals living in the low-transmission region of the Peruvian Amazon and 2), such antibody responses might have an association, either direct or indirect, with clinical immunity observed in asymptomatically parasitaemic individuals.
\end{abstract}

Methods: ELISA was used to assess antibody responses (IgG, IgG1 and $\lg G 3$ ) against recombinant $P$. falciparum invasion ligands of the EBL (EBA-175, EBA-181, EBA-140) and PfRh families (PfRh1, PfRh2a, PfRh2b, PfRh4 and PfRh5) in 45 individuals infected with $P$. falciparum from Peruvian Amazon. Individuals were classified as having symptomatic malaria $(\mathrm{N}=37)$ or asymptomatic infection $(\mathrm{N}=8)$.

Results: Antibody responses against both EBL and PfRh family proteins were significantly higher in asymptomatic compared to symptomatic individuals, demonstrating an association with clinical immunity. Significant differences in the total IgG responses were observed with EBA-175, EBA-181, PfRh2b, and MSP1 19 (as a control). IgG1 responses against EBA-181, PfRh2a and PfRh2b were significantly higher in the asymptomatic individuals. Total IgG antibody responses against PfRh1, PfRh2a, PfRh2b, PfRh5, EBA-175, EBA-181 and MSP1 19 proteins were negatively correlated with level of parasitaemia. IgG1 responses against EBA-181, PfRh2a and PfRh2b and lgG3 response for PfRh2a were also negatively correlated with parasitaemia.

Conclusions: These data suggest that falciparum malaria patients who develop clinical immunity (asymptomatic parasitaemia) in a low transmission setting such as the Peruvian Amazon have antibody responses to defined P. falciparum invasion ligand proteins higher than those found in symptomatic (non-immune) patients. While these findings will have to be confirmed by larger studies, these results are consistent with a potential role for one or more of these invasion ligands as a component of an anti-P. falciparum vaccine in low-transmission malaria-endemic regions.

Keywords: Antibodies, Invasion, Plasmodium falciparum, Malaria, Peru

\footnotetext{
* Correspondence: jvinetz@AD.UCSD.EDU; slustigman@nybloodcenter.org

${ }^{\dagger}$ Equal contributors

"Malaria Laboratory, Instituto de Medicina Tropical "Alexander von

Humboldt", Universidad Peruana Cayetano Heredia, Lima, Peru

${ }^{3}$ Departamento de Ciencias Celulares y Moleculares, Facultad de Ciencias y

Filosofia, Universidad Peruana Cayetano Heredia, Lima, Peru

Full list of author information is available at the end of the article
} 


\section{Background}

Erythrocyte invasion by Plasmodium falciparum is a complex process including attachment, reorientation, penetration, and formation of a parasitophorous vacuole. Several merozoite proteins that have a role during the initial steps of attachment and invasion have been extensively studied, including members of the Merozoite Surface Protein family (MSP), AMA-1, Erythrocyte Binding-Like proteins (EBL: EBA-175, EBA-181, EBA-140 and EBL-1), and the Reticulocyte Binding-Like or Reticulocyte Homologue proteins (RBL or PfRh: PfRh1, PfRh2a, PfRh2b, PfRh4 and PfRh5) [1]. Many of the invasion ligands are currently being evaluated or developed as candidate vaccine antigens for inclusion in an antierythrocytic-stage malaria vaccine [2]. Antibodies that inhibit merozoite attachment and invasion, and thus subsequent development and propagation within the red blood cells (RBC), are believed to be important in mediating naturally acquired immunity as well as immunity generated by parasite blood stage vaccine candidates [3]. Moreover, the cytophilic IgG1 and IgG3 antibody isotype subclasses have been reported to be associated with protective responses generated against invasion ligands [4-6], by enabling the activation of complement and antibodydependent phagocytosis and consequently parasite clearance [7]. However, it remains unclear which merozoite invasion ligand antigens might be the most important targets of naturally acquired clinical immunity, and whether the importance of such antigens are of regional specificity or globally relevance [2].

Malaria in the Amazonian region is hypoendemic and characterized by a low transmission [8]. The malaria infections are most commonly caused by Plasmodium vivax, but P. falciparum is still responsible for the major cases of severe malaria, and these infections continue to persist even though control measures are in place [9]. Previous studies in this region have demonstrated that clinical immunity to malaria is manifested by the presence of individuals with asymptomatic parasitaemia, which is not infrequent $[8,10]$. Importantly, asymptomatic parasitaemia has major implications for public health, particularly in maintaining transmission including the introduction or reintroduction of parasites in endemic regions that stopped having malaria. Understanding the immune mechanisms by which infected humans control parasitaemia in the absence of symptoms has important implications for developing anti-malarial vaccine strategies [10]. In individuals living in areas of intense $P$. falciparum transmission clinical immunity to symptomatic malaria is thought to be acquired only after repeated exposure [2]. In contrast, studies have demonstrated in Indonesia and in Amazonia that acquisition of clinical immunity can be rapid (within two years), especially in adults, and may require few infections [9-15]. This observation clearly indicates that non-sterilizing but effective clinical anti-malarial immunity develops in low transmission regions [9].

Given the epidemiological observations indicating clinical immunity against $P$. falciparum, this study aimed to test the hypothesis that antibody responses against $P$. falciparum invasion ligands belonging to both EBL and PfRh protein families might differ between symptomatic (Sym) and asymptomatic (Asy) individuals living in the low-transmission region of the Peruvian Amazon, and hence potentially contributing to explaining mechanisms of clinical immunity observed in the Asy individuals. Recombinant P. falciparum proteins corresponding to the known EBL and PfRh invasion ligands were used to determine the total IgG and IgG isotype-specific antibody responses in both study groups.

\section{Methods}

\section{Study population}

This study was approved by the Universidad Peruana Cayetano Heredia Institutional Review Board (Comite de Etica) in Lima, Peru, and by the New York Blood Center's IRB (protocol \#415). Informed consent was obtained from each adult individual or from the parents or guardians of children less than 18 years of age. The crosssectional study took place in the Peruvian Amazon region of Loreto in 2008-2010.

A total of 45 plasma samples from individuals infected with $P$. falciparum were collected over the period of 2008-2010 from 14 different communities in Loreto. The individuals were classified into two groups: 37 individuals with symptomatic malaria (16 females and 21 males; median age $=29$; range 9-63 years) and eight individuals with asymptomatic infection (one female and seven males; median age $=26$; range $12-43$ years). Asymptomatic individuals were identified by active case detection after carrying out a malaria diagnostic survey in communities that reported a malaria outbreak. Passive case detection was used to enroll symptomatic individuals who attended the local Health Care Center for diagnosis and treatment and were diagnosed with $P$. falciparum infection by light microscopy. The microscopic diagnosis in all individuals enrolled was confirmed as $P$. falciparum single infection by real time PCR [16]. Individuals were classified as Asy if they did not have any signs or symptoms related to malaria [eg. fever (temperature $>37^{\circ} \mathrm{C}$ ), chills, sweating, headache or any other kind of symptoms that the subject interpreted as indicating malaria but were positive by blood smear for P. falciparum]. Individuals were excluded if they used anti-malarial medication over the two weeks before enrollment and sample collection. No follow up was done. Blood samples were collected before all individuals were treated for malaria with mefloquine/artesunate 
following the National Drug Policy Guidelines of the Ministry of Health of Peru.

\section{Proteins used in the ELISA assays}

The following recombinant EBL and PfRh proteins corresponding to the $P$. falciparum $3 \mathrm{D} 7$ laboratory strain were used for ELISA. The C-terminal region of EBA-181 (aa 1235-1345), PfRh1 (aa 1318-1574), PfRh2a (aa 28132933), PfRh2b (aa 2735-3133) and PfRh5 (aa 31-174) were expressed in Escherichia coli BL21. PfRh2b and PfRh5 were expressed as fusion proteins with glutathione S-transferase (GST) using the pGEX 4T-3 expression vector (Amersham Biotech, NJ). The E. coli expressed PfRh4 (aa 1160-1370) protein was a kind gift from Deepak Gaur (ICGEB; New Delhi, India) and Louis Miller (NIH, USA) [17]. Fragments corresponding to the region II of EBA-175 (aa 145-760) and EBA-140 (aa 141-756) were expressed in Pichia pastoris and were a kind gift from David Narum and Louis Miller (NIH, USA) [5]. The recombinant protein corresponding to the 19-kDa processed fragment of MSP-1 (MSP1 19 ; MRA-55, MR4, ATCC Manassas Virginia) was also used as a control [18].

\section{IgG responses against $P$. falciparum invasion ligands as determined by ELISA}

Peruvian plasma samples were analyzed for total IgG, IgG1 and IgG3 reactivity to the recombinant EBL and PfRh ligands by ELISA as previously described [19]. Plasma samples were pre-incubated with $E$. coli extract prior to dilution and incubation with the bound antigen to remove antibodies to potential $E$. coli contaminants in the recombinant protein preparations. Briefly, 96-well ELISA plates (Costar, Corning Life Sciences, Corning, $\mathrm{NY}$ ) were coated with $1 \mu \mathrm{g} / \mathrm{mL}$ of the recombinant protein $(0.5 \mu \mathrm{g} / \mathrm{mL}$ for PfRh4) in $0.05 \mathrm{M}$ carbonate buffer, $\mathrm{pH} 9.6$ overnight at $4^{\circ} \mathrm{C}$. The next day, plates were washed 6 times with phosphate-buffered saline (PBS) containing $0.05 \%$ Tween 20 (PBST) and blocked for 1.5-2 h with blocking buffer ( $3 \% \mathrm{BSA}$ in PBST) at $37^{\circ} \mathrm{C}$. Plates were washed with PBST and test and control plasma samples (1:200) diluted in blocking buffer were added into each well in duplicate for $2 \mathrm{~h}$ at $37^{\circ} \mathrm{C}$. The plates were then washed 6 times with PBST and the bound IgG antibodies were detected after $1 \mathrm{~h}$ of incubation at $37^{\circ} \mathrm{C}$ using HRP-conjugated goat anti-human $(1: 1,000)$ IgG $(\mathrm{H}+\mathrm{L}$; Kirkegaard and Perry Laboratories, Inc., Gaithersburg, MD). IgG1 and IgG3 antibodies were detected after incubation for $1 \mathrm{hr}$ at $37^{\circ} \mathrm{C}$ with mouse monoclonal antibodies against human IgG1 or IgG3 (Hybridoma Reagent Laboratory, Baltimore, MD) diluted 1:1,000 in blocking buffer, followed by HRP-conjugated anti-mouse (1:1250). After 6X washing with PBST, bound antibodies were detected by adding the SureBlue tetramethyl benzidine (TMB) substrate (Kirkegaard and Perry Laboratories, Inc., Gaithersburg, MD). The reaction was stopped by the addition of $2 \mathrm{~N} \mathrm{H}_{2} \mathrm{SO}_{4}$, and the Optical Density (OD) at $450 \mathrm{~nm}$ was measured using a SpectraMax 190 ELISA Reader (Molecular Devices, Sunnyvale, CA). For the GST-fusion PfRh2b and PfRh5 proteins, each plasma sample was also tested against recombinant GST as a negative control and the corresponding absorbance was deducted from all test values for the fusion proteins. The negative control human serum was composed of a pool of sera from five healthy adult volunteers living in USA who had never been exposed to malaria. Positive responders were defined as those that gave an OD value greater than the mean plus three standard deviations (SD) of the reactivity observed with negative control serum (cut-off value).

\section{Statistical analysis}

Fisher's exact test ( $2 \times 2$ contingency tables) was used to compare differences in the seroprevalence between groups. The Mann-Whitney test was used to compare medians between different groups. Correlations between antibody response (as indicated by OD) and parasitaemia or age were examined using Spearman's test. A $p$ value $<0.05$ was considered statistically significant. Statistical analyses were performed using GraphPad Prism software (version 5.01; GraphPad Software Inc., San Diego, CA, USA).

\section{Results}

\section{Differential antibody responses in asymptomatic vs symptomatic individuals}

Two groups of individuals were studied; individuals with symptomatic malaria (Sym, $\mathrm{N}=37$ ) and individuals with asymptomatic infection (Asy, $\mathrm{N}=8$ ). There were no significant differences in the sex distribution or median of age between the groups. Parasitaemia was lower in the Asy (median 519 parasites $/ \mu \mathrm{L}$; range 24-2874 parasites $/ \mu \mathrm{L}$ ) than in the Sym individuals (median 7056 parasites $/ \mu \mathrm{L}$; range $239-61,185$ parasites $/ \mu \mathrm{L})(p<0.0001)$.

Samples were tested against a comprehensive panel of known EBL and PfRh ligands except EBL-1; a majority of the Peruvian P. falciparum field isolates do not express EBL-1 as the gene in these parasites contained a fivethymidine insertion that results in premature translational termination and lack of protein expression [Lopez-Perez $\mathrm{M}$ et al., manuscript in preparation]. Antibodies against $\mathrm{MSP}_{19}$, an immunogenic merozoite protein targeted as a vaccine protein, were measured for comparison. The percentage of responders against this antigen was $100 \%$ in Asy vs. 54\% in the Sym (Table 1). Based on the total IgG responses against the EBL and PfRh invasion ligands, the percentage of responders in the Asy vs. the Sym group varied for some. In general, a higher seroprevalence was 
Table 1 Percentages of individuals within the Asymptomatic (Asy) and Symptomatic (Sym) malaria groups responding (total lgG) to EBL and PfRh ligands

\begin{tabular}{|c|c|c|c|c|c|c|c|c|c|}
\hline Ligand & PfRh1 & PfRh2a & PfRh2b & PfRh4 & PfRh5 & EBA- 175 & EBA- 140 & EBA-181 & $\mathrm{MSP} \mathbf{1}_{19}$ \\
\hline Asy & $100 \%$ & $75 \%$ & $75 \%$ & $71 \%$ & $25 \%$ & $100 \%$ & $25 \%$ & $88 \%$ & $100 \%$ \\
\hline Seropositive & $(8 / 8)$ & $(6 / 8)$ & $(6 / 8)$ & $(5 / 7)$ & $(2 / 8)$ & $(8 / 8)$ & $(2 / 8)$ & $(7 / 8)$ & $(8 / 8)$ \\
\hline Sym & $86.5 \%$ & $75.7 \%$ & $21.6 \%$ & $47 \%$ & $8.1 \%$ & $32.4 \%$ & $5.4 \%$ & $21.6 \%$ & $54 \%$ \\
\hline Seropositive & $(32 / 37)$ & $(28 / 37)$ & $(8 / 37)$ & $(17 / 36)$ & $(3 / 37)$ & $(12 / 37)$ & $(2 / 37)$ & $(8 / 37)$ & $(20 / 37)$ \\
\hline Cut-off value & 0.13 & 0.12 & 0.1 & 0.1 & 0.16 & 0.16 & 0.41 & 0.12 & 0.16 \\
\hline$p$ (Fisher Test) & ns & ns & 0.007 & ns & ns & 0.0006 & ns & 0.0009 & 0.0171 \\
\hline
\end{tabular}

observed in Asy individuals against EBA-175, EBA-181, PfRh1, PfRh2b and PfRh4 than in Sym individuals. The percentage of responders against PfRh2a was similar (75\%). Notably, there were fewer responders in both groups against EBA-140 (25\% and 5.4\%) and PfRh5 (25\% and $8.1 \%$ ) (Table 1). When the seroprevalence between both groups for each invasion ligand was compared, it appeared that the percentage of responders in the Asy individuals was significantly higher than in the Sym group only against EBA-175 $(p=0.0006)$, EBA-181 $(p=0.0009)$ and PfRh2b $(p=0.007)$ as well as against the control merozoite protein, $\mathrm{MSP}_{19}(p=0.0171)$. Significantly, a higher total IgG response, as indicated by the OD value, was observed in Asy than Sym individuals for EBA175 (median OD 1.08 vs. 0.15; $p=0.0001$ ), EBA-181 (median OD 0.50 vs. $0.11 ; p=0.0004$ ), PfRh1 (median OD 0.23 vs. $0.16 ; p=0.0006$ ) and PfRh2b (median OD 0.36 vs. $0.01 ; p=0.0022$ ) as well as for $\mathrm{MSP}_{19}$ (median OD 0.90 vs. $0.17 ; p=0.0005$ ) (Figure 1 ).

Total IgG and isotype responses against some of the invasion ligands are also associated with reduced parasitaemia

As Asy individuals had significantly lower parasitaemia than Sym, ELISA was used to determine whether IgG levels, as indicated by optical density, were also negatively associated with parasitaemia (parasites $/ \mu \mathrm{L}$ ). A significant negative correlation was observed between the total IgG levels and parasitaemia for the following invasion ligands: PfRh1 $\left(p<0.0001, \mathrm{r}_{\mathrm{s}}=-0.565\right)$, PfRh2a $(p=0.007$, $\left.\mathrm{r}_{\mathrm{s}}=-0.499\right)$, PfRh2b $\left(p=0.0004, \mathrm{r}_{\mathrm{s}}=-0.515\right)$, PfRh5 $\left(p=0.016, \mathrm{r}_{\mathrm{s}}=-0.364\right)$, EBA-175 $\left(p=0.0004, \mathrm{r}_{\mathrm{s}}=-0.516\right)$, EBA-181 $\left(p=0.0001, \mathrm{r}_{\mathrm{s}}=-0.546\right)$ as well as for $\mathrm{MSP}_{19}\left(p<0.00001, \mathrm{r}_{\mathrm{s}}=-0.619\right)$. The total IgG responses against EBA-140 and PfRh4 ligands were not associated with levels of parasitaemia (Figure 2).

$\lg \mathrm{G} 1$ and $\lg G 3$ isotype-specific antibody responses to the EBL and PfRh ligands

IgG1 and IgG3 are the predominant isotypes produced in response to merozoite invasion ligands [4-6], and these isotypes are associated with being cytophilic and mechanistically killing parasitized RBCs by antibodydependent cell-mediated inhibition (ADCI) [7]. Thus, to gain additional insights in the differential antibody response generated in the Asy and Sym individuals under natural infection conditions, we measured IgG1 and IgG3 responses for PfRh2a, PfRh2b, PfRh5 and EBA-181, for which a higher total IgG response was observed to be negatively correlated with lower

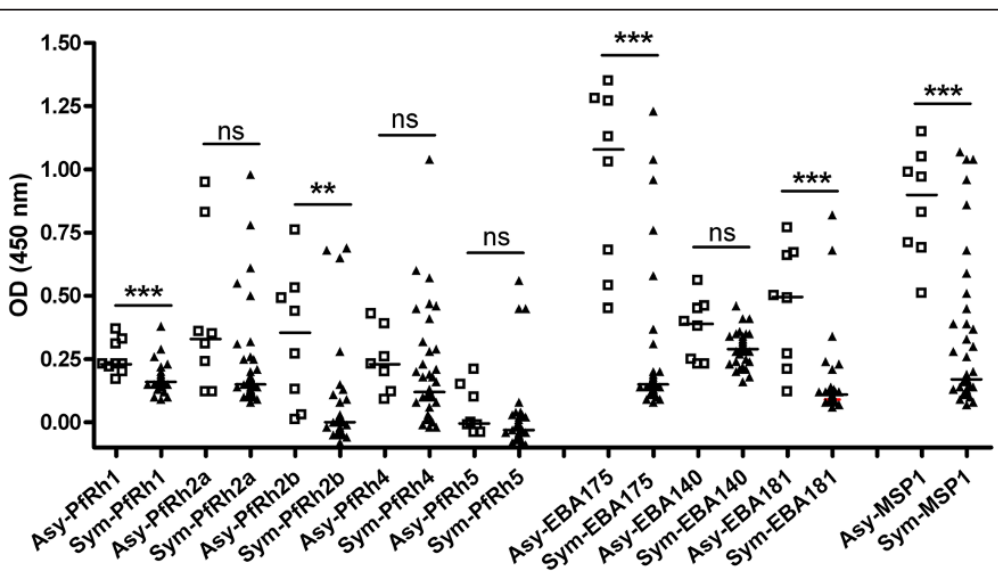

Figure 1 Total IgG responses to EBL and PfRh ligands in Symptomatic and Asymptomatic Peruvian individuals. Comparison between the total lgG responses (as indicated by optical density, OD) in asymptomatic (Asy) and symptomatic (Sym) Peruvian individuals. Median values are indicated by horizontal bars. Abbreviations: Asy, open symbols; Sym, close symbols; ns, not significant; ${ }^{* *} p<0.01$; ${ }^{* *} p<0.001$. 


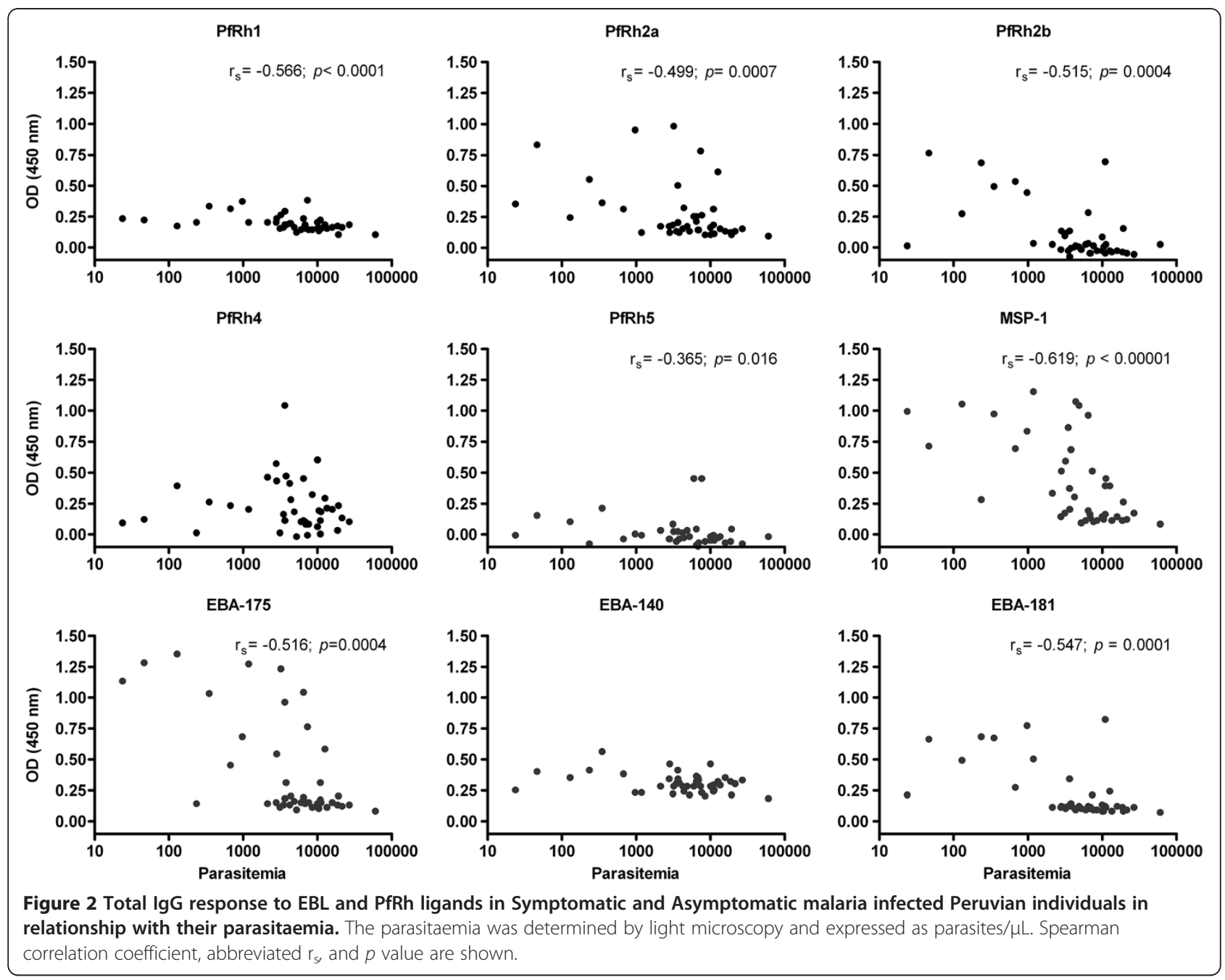

parasitaemia (Figure 2). A higher IgG1 seroprevalence was observed in the Asy than the Sym group against EBA-181 (88\% vs. 24\%; $p=0.002)$, PfRh2a ( $88 \%$ vs. 38\%; $p=0.017)$ and PfRh2b (88\% vs. $24 \% ; p=0.003)$. The prevalence of IgG3 responders was higher in Asy than Sym for PfRh2a (75\% vs. 30\%; $p=0.039)$ and PfRh5 (38\% vs. $3 \% ; p=0.014)$.

Differences in IgG1 and IgG3 responses between the Asy land Sym individuals were also observed (Figure 3). gG1 responses for EBA-181 (median OD 0.46 vs. 0.09; $p=0.0006$ ) and PfRh2b (median OD $0.36 v s$. 0.07; $p=0.0017$ ) were significantly higher in the Asy than the Sym group. Notably, both IgG1 and IgG3 were dominant in the Asy group for PfRh2a (median IgG1 OD 0.70 vs. $0.19 ; p=0.0018$ and median IgG3 OD 0.34 vs. 0.2 ; $p=0.0206)$.

Notably, IgG1 and IgG3 levels were also significantly associated with lower parasitaemia (Figure 4). A negative correlation was observed between parasitaemia and the IgG1 levels for EBA-181 $\left(p=0.0007 ; r_{\mathrm{s}}=-0.495\right)$,
$\operatorname{PfRh} 2 \mathrm{a}\left(p=0.0178 ; \mathrm{r}_{\mathrm{s}}=-0.360\right)$ and PfRh2b $(p=0.015$; $\left.\mathrm{r}_{\mathrm{s}}=-0.368\right)$, and the IgG3 levels for PfRh2a $(p=0.0028$; $\left.r_{\mathrm{s}}=-0.445\right)$.

\section{Discussion}

This study is the first to comprehensively analyse the profile of naturally acquired antibodies against the two major families of $P$. falciparum merozoite ligands in asymptomatic $v s$ symptomatic populations living in a malaria hypoendemic area. Sera from P. falciparum-infected individuals from the Peruvian Amazon were tested against all PfRh and EBL family members known to be involved in $P$. falciparum invasion. This study's main finding is that antibody responses against the EBL and PfRh proteins were significantly higher in asymptomatic than symptomatic parasitaemic individuals, suggesting a potential association with the development of clinical immunity. Whether these anti-invasion ligand antibodies directly mediate protective immunity or are simply statistically associated with clinical immunity is beyond the scope of this pilot 


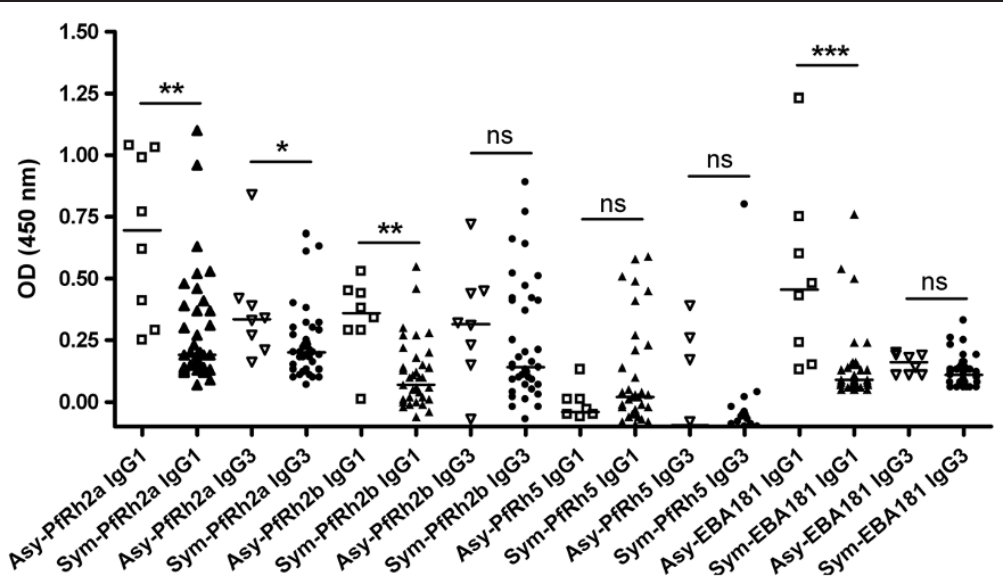

Figure 3 Comparison of $\operatorname{lgG} 1$ and $\lg \mathrm{G} 3$ isotypes-specific antibody response to EBA-181 and PfRh ligands in asymptomatic and symptomatic Peruvian individuals. Comparison between the lgG1 and lgG3 responses (as indicated by optical density, OD) against some of the invasion ligands in asymptomatic (Asy) and symptomatic (Sym) Peruvian individuals. Median values are indicated by horizontal bars. Abbreviations: Asy, open symbols; Sym, close symbols; ns, not significant; ${ }^{*} p<0.05$; ${ }^{*} p<0.01$; ${ }^{* *} p<0.001$.

study. Significant differences in the total IgG responses were observed against EBA-175, EBA-181, PfRh2b and $\mathrm{MSP}_{19}$. IgG1 responses against EBA-181, PfRh2a and PfRh2b were also significantly higher in the asymptomatic individuals. Furthermore, elevated total IgG antibody responses against PfRh1, PfRh2a, PfRh2b, PfRh5, EBA$175, \mathrm{EBA}-181$ and $\mathrm{MSP}_{19}$ proteins were positively associated with decreased parasitaemia. IgG1 response against EBA-181, PfRh2a and PfRh2b and IgG3 response for $\mathrm{PfRh} 2 \mathrm{a}$ were also negatively correlated with parasitaemia. Thus, these results suggest that total and IgG subclassspecific responses to distinct merozoite antigens are significantly associated with protection from high-density parasitaemia and symptomatic malaria.

IgG responses to some but not all EBL antigens has been associated with protection against symptomatic $P$. falciparum in some malaria endemic areas (reviewed in [1]). Naturally acquired antibodies to region II of EBA-175 have been found to increase with age in a naturally exposed population in Kenya and such antibodies were capable of inhibiting binding of EBA-175RII to erythrocytes as a correlate of clinical immunity in this population affected by holoendemic malaria [20]. Nonetheless, significant associations between anti-EBA-175 antibody levels and protection from high-density parasitaemia and clinical disease has been inconsistent among studies [20-23] although experimental studies using sera from rabbits vaccinated with region II of EBA-175 were able to block invasion by $>50 \%$ of $P$. falciparum laboratory strains from diverse geographic origin [24,25]. Studies that measure responses to the other EBL antigens in endemic areas outside of Africa are scarcer $[5,9,19]$. A comparative study by Ford et al., showed that the antibody responses against MSP $1_{19}$ and EBLs (EBA-175, EBA-140, EBA-181) in individuals living in a hypoendemic malaria region of Brazil were much lower thanin individuals living in a hyperendemic area of Cameroon [19].

Immune responses against PfRh invasion ligands have been less studied [4,26,27]. Association between antibody responses against PfRh ligands and outcome of a clinical disease has been reported only for $\mathrm{PfRh} 2 \mathrm{a}$ and PfRh2b [4]. Antibody responses against PfRh4, PfRh5 and the common region of PfRh2 were reported for subjects in Kenya without taking into consideration the clinical or parasitological status of the studied individuals [27], and responses against PfRh2b was also evaluated in subjects from Senegal and Tanzania [26]. In children from Papua New Guinea, high levels of total IgG as well as IgG1 and IgG3 antibody responses against the $\mathrm{PfRh} 2 \mathrm{a}$ and PfRh2b antigens were found to be strongly associated with protection from symptomatic malaria and high-density parasitaemia. Notably, these antibody responses were similar to or greater than the ones generated against the leading vaccines candidates $M S P 1_{19}$, MSP-2 and AMA-1 [4], but, it must be noted, that different regions of the EBAs and the PfRH proteins were studied so it may be difficult to make direct comparisons [4]. Antibody responses against PfPh5 have been found at much lower rates in naturally infected individuals but laboratory studies of anti-PfRh 5 antibodies induced by vaccination with recombinant protein appeared to induce strain-transcending growth inhibitory antibodies [25,27]. The focus on PfRh invasion ligands as potential vaccine antigens was augmented by the recent promising invasion inhibitory results obtained using antibodies generated against the combination of EBL and PfRh proteins; EBA-175, PfRh2 and PfRh4 [28], and EBA-175 and PfRh5 [25]. The ability of antibodies against the full length PfRh5 (RH5FL) protein to inhibit the growth of all strains 


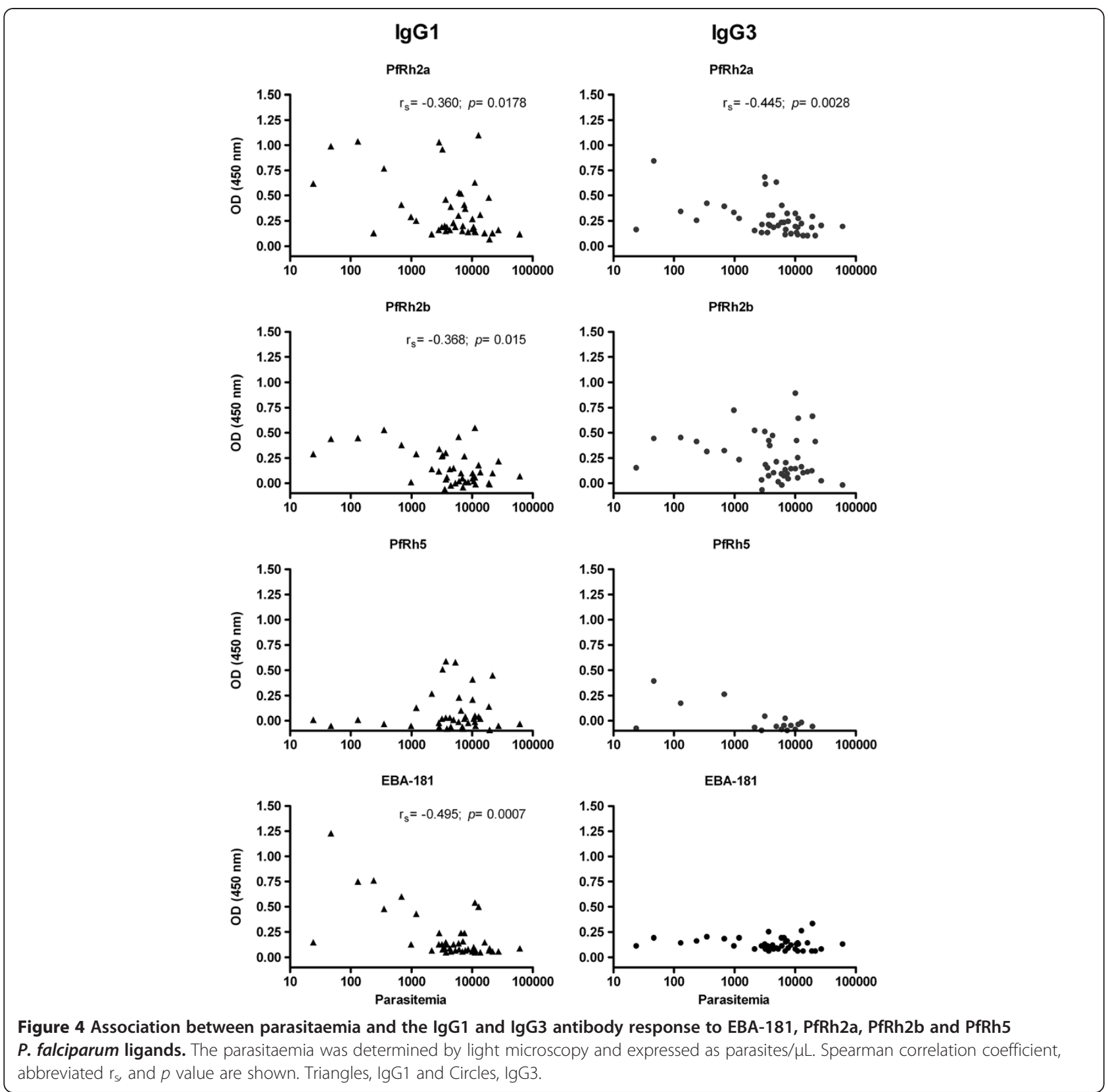

of parasites in vitro more effectively than antibodies induced by other vaccine candidates, such as AMA-1 and MSP-1, increased the appeal of using PfRh5 as an anti-blood-stage malaria vaccine [27].

Three studies have previously examined the presence of antibodies against PfRh ligands [4,26,27], but not against PfRh1 in an endemic population. Notably, total IgG response to PfRh1 in the Peruvian population was similar in Sym and Asy individuals based on seroprevalence, but the magnitude of the response was significantly higher in the Asy group and inversely associated with levels of parasitaemia. A high seroprevalence for PfRh2a (75\%) regardless of the clinical status (Asy and
Sym) was observed in the Peruvian individuals similar to what was reported in children from Papua New Guinea (94\%) [4]. However, in Peruvian subjects, significant differences were observed in the prevalence of IgG1 and IgG3 responders in the Asy group (IgG1 88\% vs. 38\% and IgG3 75\% vs. 30\%). In contrast, the response against PfRh2b in the Peruvians (31\%) was higher than that reported in individuals with malaria in Senegal (8.9\%) and Tanzania (5.6\%) and lower than those observed in Papua New Guinea (85\%) [4,26]. However, higher total IgG and IgG1 responses against the PfRh2b ligand were associated with low parasitaemia, similar to observed in Papua New Guinea [4]. Seroprevalence for PfRh4 in the 
Peruvian infected individuals was lower than observed in adults from Kenya (50\% vs. 70\%) [27], and presently it appears that these antigen specific responses are not associated with protection against clinical malaria. In this study only IgG1 and IgG3 subtypes were measured and analysed because these subtypes are associated with $\mathrm{F}_{\mathrm{c}}$ binding and are potentially associated with functional antibody-dependent cellular cytotoxicity $[29,30]$. Nonetheless, whether such anti-invasion ligand antibodies might function through $\mathrm{ADCI}$, steric hindering or by another mechanism remains to be determined. Although PfRh5 was suggested to be essential for erythrocyte invasion $[31,32]$ and the target for vaccine-induced antibodies [27], only few individuals from Peru had antibodies against PfRh5 (11\%), which is comparable to what was reported in Kenya (16\%) [27]. The reason for the lack of anti-PfRh 5 antibody in naturally exposed individuals is still unclear. The mechanism by which this essential invasion ligand escapes immune recognition thus requires further investigation.

\section{Conclusions}

Although the contribution of the anti-invasion EBL and/or PfRh ligand antibody responses to protective immunity needs to be confirmed in future studies by functional assays such as growth inhibition assays, the present study strongly suggests that the Peruvian population present in a low transmission setting has a capacity to develop clinical immunity that is likely associated with antibody responses to defined $P$. falciparum invasion ligands. These results are consistent with a potential role for one or more of these invasion ligands as a component of an anti- $P$. falciparum vaccine in the low-transmission areas of the Amazon region, but may argue against a one-size fits all strain-transcending blood stage malaria vaccine.

\section{Abbreviations \\ EBL: Erythrocyte binding-like; PfRh: Reticulocyte binding-like; OD: Optical density; Asy: Asymptomatic; Sym: Symptomatic; ADCl: Antibody-dependent cell-mediated inhibition.}

\section{Competing interests}

The authors declare that they have no competing interests.

\section{Authors' contributions}

$K T, D G, J M V$ and SL participated in the design of the study. EV, KT, VN, JB collected the samples. MLP, NT and CL designed or carried out the ELISA studies. EV, MLP, JMV and SL drafted the manuscript. All authors read and approved the final manuscript.

\section{Acknowledgments}

We thank Marilis Rodriguez, Blood-Borne Parasites, New York Blood Center, for the production of the recombinant ligands we used in this study. We thank Deepak Gaur [International Centre for Genetic Engineering and Biotechnology (ICGEB) New Delhi, India] and Louis Miller (NIH, USA) for kindly providing recombinant protein PfRh4. We thank David Narum and Louis Miller from (NIH, USA) for kindly providing recombinant proteins EBA175 and EBA-140. The following reagent was obtained through the MR4 as part of the BEI Resources Repository, NIAID, NIH: Plasmodium falciparum
yPfMSP1-19(Q-TSR)VK1, MRA-55, deposited by DC Kaslow. The authors are also very grateful to the local health personnel in all sites of this study and the participating patients. We thank Paula Maguina, UC San Diego for scientific and logistical contributions essential to carrying out this study. This work was supported by the Peru/Brazil International Center of Excellence in Malaria Research U19AI089681 (NIH/NIAID, United States Public Health Service, USA), the NIH/Fogarty International Center Global Infectious Diseases Training Grant D43TW007120 (IMTAvH-UCSD), and the New York Blood Center.

\section{Author details}

'Malaria Laboratory, Instituto de Medicina Tropical "Alexander von Humboldt", Universidad Peruana Cayetano Heredia, Lima, Peru. 'Molecular Parasitology, Lindsley F. Kimball Research Institute, New York Blood Center, NYC, New York, NY, USA. ${ }^{3}$ Departamento de Ciencias Celulares y Moleculares, Facultad de Ciencias y Filosofia, Universidad Peruana Cayetano Heredia, Lima, Peru. ${ }^{4}$ Blood-Borne Parasites, Lindsley F. Kimball Research Institute, New York Blood Center, NYC, New York, NY, USA. ${ }^{5}$ Division of Infectious Diseases,

Department of Medicine, University of California San Diego, La Jolla, CA, USA.

Received: 20 July 2012 Accepted: 25 October 2012

Published: 30 October 2012

\section{References}

1. Tham WH, Healer J, Cowman AF: Erythrocyte and reticulocyte binding-like proteins of Plasmodium falciparum. Trends Parasitol 2012, 28:23-30.

2. Fowkes FJ, Richards JS, Simpson JA, Beeson JG: The relationship between anti-merozoite antibodies and incidence of Plasmodium falciparum malaria: a systematic review and meta-analysis. PLoS Med 2010, 7:e1000218.

3. Persson KE: Erythrocyte invasion and functionally inhibitory antibodies in Plasmodium falciparum malaria. Acta Trop 2010, 114:138-143.

4. Reiling L, Richards JS, Fowkes FJ, Barry AE, Triglia T, Chokejindachai W, Michon P, Tavul L, Siba PM, Cowman AF, Mueller I, Beeson JG: Evidence that the erythrocyte invasion ligand PfRh2 is a target of protective immunity against Plasmodium falciparum malaria. J Immunol 2010, 185:6157-6167.

5. Richards JS, Stanisic DI, Fowkes FJ, Tavul L, Dabod E, Thompson JK, Kumar S, Chitnis CE, Narum DL, Michon P, Siba PM, Cowman AF, Mueller I, Beeson JG: Association between naturally acquired antibodies to erythrocytebinding antigens of Plasmodium falciparum and protection from malaria and high-density parasitemia. Clin Infect Dis 2010, 51:e50-e60.

6. Stanisic DI, Richards JS, McCallum FJ, Michon P, King CL, Schoepflin S, Gilson PR, Murphy VJ, Anders RF, Mueller I, Beeson JG: Immunoglobulin G subclass-specific responses against Plasmodium falciparum merozoite antigens are associated with control of parasitemia and protection from symptomatic illness. Infect Immun 2009, 77:1165-1174.

7. Jafarshad A, Dziegiel MH, Lundquist R, Nielsen LK, Singh S, Druilhe PL: A novel antibody-dependent cellular cytotoxicity mechanism involved in defense against malaria requires costimulation of monocytes FcgammaRII and FcgammaRIII. J Immunol 2007, 178:3099-3106.

8. da Silva-Nunes M, Moreno M, Conn JE, Gamboa D, Abeles S, Vinetz JM, Ferreira MU: Amazonian malaria: asymptomatic human reservoirs, diagnostic challenges, environmentally driven changes in mosquito vector populations, and the mandate for sustainable control strategies. Acta Trop 2012, 121:281-291.

9. Clark EH, Silva CJ, Weiss GE, Li S, Padilla C, Crompton PD, Hernandez JN, Branch OH: Plasmodium falciparum malaria in the Peruvian Amazon, a region of low transmission, is associated with immunologic memory. Infect Immun 2012, 80:1583-1592.

10. Vinetz JM, Gilman RH: Asymptomatic Plasmodium parasitemia and the ecology of malaria transmission. Am J Trop Med Hyg 2002, 66:639-640.

11. Jordan SJ, Oliveira AL, Hernandez JN, Oster RA, Chattopadhyay D, Branch $\mathrm{OH}$, Rayner JC: Malaria immunoepidemiology in low transmission: correlation of infecting genotype and immune response to domains of Plasmodium falciparum merozoite surface protein 3. Infect Immun 2011, 79:2070-2078,

12. Alves FP, Durlacher RR, Menezes MJ, Krieger H, Silva LH, Camargo EP: High prevalence of asymptomatic Plasmodium vivax and Plasmodium falciparum infections in native Amazonian populations. Am J Trop Med Hyg 2002, 66:641-648. 
13. Camargo EP, Alves F, da Silva Pereira LH: Symptomless Plasmodium vivax infections in native Amazonians. Lancet 1999, 353:1415-1416.

14. Roshanravan B, Kari E, Gilman RH, Cabrera L, Lee E, Metcalfe J, Calderon M, Lescano AG, Montenegro SH, Calampa C, Vinetz JM: Endemic malaria in the Peruvian Amazon region of Iquitos. Am J Trop Med Hyg 2003, 69:45-52.

15. Sutton PL, Clark EH, Silva C, Branch OH: The Plasmodium falciparum merozoite surface protein-1 19 KD antibody response in the Peruvian Amazon predominantly targets the non-allele specific, shared sites of this antigen. Malar J 2010, 9:3.

16. Mangold KA, Manson RU, Koay ES, Stephens L, Regner M, Thomson RB Jr, Peterson LR, Kaul KL: Real-time PCR for detection and identification of Plasmodium spp. J Clin Microbiol 2005, 43:2435-2440.

17. Gaur D, Singh S, Jiang L, Diouf A, Miller LH: Recombinant Plasmodium falciparum reticulocyte homology protein 4 binds to erythrocytes and blocks invasion. Proc Natl Acad Sci U S A 2007, 104:17789-17794.

18. Kaslow DC, Hui G, Kumar S: Expression and antigenicity of Plasmodium falciparum major merozoite surface protein (MSP1(19)) variants secreted from Saccharomyces cerevisiae. Mol Biochem Parasitol 1994, 63:283-289.

19. Ford L, Lobo CA, Rodriguez M, Zalis MG, Machado RL, Rossit AR, Cavasini CE, Couto AA, Enyong PA, Lustigman S: Differential antibody responses to Plasmodium falciparum invasion ligand proteins in individuals living in malaria-endemic areas in Brazil and Cameroon. Am J Trop Med Hyg 2007, 77:977-983.

20. Ohas EA, Adams JH, Waitumbi JN, Orago AS, Barbosa A, Lanar DE, Stoute JA: Measurement of antibody levels against region II of the erythrocytebinding antigen 175 of Plasmodium falciparum in an area of malaria holoendemicity in western Kenya. Infect Immun 2004, 72:735-741.

21. John CC, Moormann AM, Pregibon DC, Sumba PO, McHugh MM, Narum DL, Lanar DE, Schluchter MD, Kazura JW: Correlation of high levels of antibodies to multiple pre-erythrocytic Plasmodium falciparum antigens and protection from infection. Am J Trop Med Hyg 2005, 73:222-228.

22. Okenu DM, Riley EM, Bickle QD, Agomo PU, Barbosa A, Daugherty JR, Lanar $D E$, Conway DJ: Analysis of human antibodies to erythrocyte binding antigen 175 of Plasmodium falciparum. Infect Immun 2000, 68:5559-5566.

23. Osier FH, Fegan G, Polley SD, Murungi L, Verra F, Tetteh KK, Lowe B, Mwangi T, Bull PC, Thomas AW, Cavanagh DR, McBride JS, Lanar DE, Mackinnon MJ, Conway DJ, Marsh K: Breadth and magnitude of antibody responses to multiple Plasmodium falciparum merozoite antigens are associated with protection from clinical malaria. Infect Immun 2008, $76: 2240-2248$.

24. Jiang L, Gaur D, Mu J, Zhou H, Long CA, Miller LH: Evidence for erythrocyte-binding antigen 175 as a component of a ligand-blocking blood-stage malaria vaccine. Proc Natl Acad Sci U S A 2011, 108:7553-7558.

25. Ord RL, Rodriguez M, Yamasaki T, Takeo S, Tsuboi T, Lobo CA: Targeting sialic acid dependent and independent pathways of invasion in Plasmodium falciparum. PLoS One 2012, 7:e30251.

26. Ahouidi AD, Bei AK, Neafsey DE, Sarr O, Volkman S, Milner D, Cox-Singh J, Ferreira MU, Ndir O, Premji Z, Mboup S, Duraisingh MT: Population genetic analysis of large sequence polymorphisms in Plasmodium falciparum blood-stage antigens. Infect Genet Evol 2010, 10:200-206.

27. Douglas AD, Williams AR, Illingworth JJ, Kamuyu G, Biswas S, Goodman AL, Wyllie DH, Crosnier C, Miura K, Wright GJ, Long CA, Osier FH, Marsh K, Turner AV, Hill AV, Draper SJ: The blood-stage malaria antigen PfRH5 is susceptible to vaccine-inducible cross-strain neutralizing antibody. Nat Commun 2011, 2:601.

28. Lopaticki S, Maier AG, Thompson J, Wilson DW, Tham WH, Triglia T, Gout A, Speed TP, Beeson JG, Healer J, Cowman AF: Reticulocyte and erythrocyte binding-like proteins function cooperatively in invasion of human erythrocytes by malaria parasites. Infect Immun 2011, 79:1107-1117.

29. Pleass RJ, Woof JM: Fc receptors and immunity to parasites. Trends Parasitol 2001, 17:545-551.

30. Roussilhon C, Oeuvray C, Muller-Graf C, Tall A, Rogier C, Trape JF, Theisen M, Balde A, Perignon JL, Druilhe P: Long-term clinical protection from falciparum malaria is strongly associated with $\lg \mathrm{G} 3$ antibodies to merozoite surface protein 3. PLoS Med 2007, 4:e320

31. Baum J, Chen L, Healer J, Lopaticki S, Boyle M, Triglia T, Ehlgen F, Ralph SA, Beeson JG, Cowman AF: Reticulocyte-binding protein homologue 5 - an essential adhesin involved in invasion of human erythrocytes by Plasmodium falciparum. Int J Parasitol 2009, 39:371-380.
32. Crosnier C, Bustamante LY, Bartholdson SJ, Bei AK, Theron M, Uchikawa M, Mboup S, Ndir O, Kwiatkowski DP, Duraisingh MT, et al: Basigin is a receptor essential for erythrocyte invasion by Plasmodium falciparum. Nature 2011, 480:534-537.

doi:10.1186/1475-2875-11-361

Cite this article as: Villasis et al:: Anti-Plasmodium falciparum invasion ligand antibodies in a low malaria transmission region, Loreto, Peru. Malaria Journal 2012 11:361.

\section{Submit your next manuscript to BioMed Central and take full advantage of:}

- Convenient online submission

- Thorough peer review

- No space constraints or color figure charges

- Immediate publication on acceptance

- Inclusion in PubMed, CAS, Scopus and Google Scholar

- Research which is freely available for redistribution 